\title{
African American/Black and Latino Adults with Detectable HIV Viral Load Evidence Substantial Risk for Polysubstance Substance Use and Co-occurring Problems: A Latent Class Analysis
}

\author{
Charles M. Cleland ${ }^{1,2} \cdot$ Marya Gwadz $^{2,3}$ - Linda M. Collins ${ }^{2,4} \cdot$ Leo Wilton $^{5,6} \cdot$ Dawa Sherpa $^{3} \cdot$ Caroline Dorsen $^{7}$. \\ Noelle R. Leonard ${ }^{2,3} \cdot$ Sabrina R. Cluesman ${ }^{3} \cdot$ Belkis Y. Martinez ${ }^{3} \cdot$ Amanda S. Ritchie $^{3} \cdot$ Mariam Ayvazyan $^{3}$
}

Accepted: 24 February 2021 / Published online: 8 March 2021

(c) The Author(s), under exclusive licence to Springer Science+Business Media, LLC, part of Springer Nature 2021

\begin{abstract}
Substance use problems are highly prevalent among persons living with (PLWH) in the United States and serve as serious barriers to engagement in HIV care. Yet, in contrast to studies of single substances, little is known about patterns of polysubstance use in this population. Moreover, other risk factors (e.g., financial hardship, incarceration, homelessness, and mental health distress) are also prevalent and complicate HIV management. The present study drew on a cross-sectional survey with African American/Black and Latino (AABL) adult PLWH from low socioeconomic status backgrounds in New York City who were insufficiently engaged in HIV care and evidenced detectable HIV viral load $(N=512)$. We used latent class analysis (LCA) to explore patterns of polysubstance use and their relationships to financial hardship, incarceration, homelessness, and mental health. LCA yielded three substance use classes: Class 1, a high polysubstance use/high-risk substance use class (9\%); Class 2, a polysubstance use/moderate substance use risk class (18\%); and Class 3, a moderate polysubstance use/moderate-to-low-risk substance use class (74\%). Mental health symptoms were prevalent in all classes, but Class 1 had greater mental health distress than the other two classes. Current homelessness was more prevalent in Classes 1 and 2. We cannot end the HIV epidemic without engaging and treating AABL PLWH who have serious barriers to engagement along the HIV care continuum, and who evidence polysubstance use along with co-occurring risk factors. Clinical settings can develop outreach and engagement approaches to bring this subpopulation of PLWH into care settings, and further, specialized services are needed to successfully screen, treat, and retain them.
\end{abstract}

Keywords HIV $\cdot$ Latent class analysis $\cdot$ Polysubstance use $\cdot$ Mental health $\cdot$ Barriers to the HIV care continuum

Marya Gwadz

Mg2890@nyu.edu

1 Division of Biostatistics, Department of Population Health, New York University Grossman School of Medicine, New York, NY, USA

2 Center for Drug Use and HIV Research, School of Global Public Health, New York University, New York, NY, USA

3 Silver School of Social Work, New York University, 1 Washington Square North, Room 303, New York, NY, USA

4 Departments of Social and Behavioral Sciences and Biostatistics, School of Global Public Health, New York University, New York, NY, USA

5 Department of Human Development, State University of New York At Binghamton, Binghamton, NY, USA

6 Faculty of Humanities, University of Johannesburg, Johannesburg, South Africa

7 Rutgers University School of Nursing, Newark, NJ, USA

\section{Introduction}

Among the population of 1.1 million persons living with HIV (PLWH) in the United States, persons from African American or Black and Latino (AABL) and low-socioeconomic status (SES) backgrounds are substantially overrepresented compared to their proportions in the general population [1]. Rates of engagement along the HIV care continuum have improved significantly in the past decade [1], but low-SES AABL PLWH are more likely to experience impediments to consistent HIV care engagement, resulting in reduced odds of persistent antiretroviral therapy (ART) use and sustained HIV viral suppression, as well as higher mortality rates, relative to their White and more socio-economically advantaged peers [2-5]. These racial/ethnic health disparities are driven by barriers and risk factors that operate simultaneously at structural, organizational, social, and 
individual levels of influence [6, 7]. For example, low SES creates complex competing priorities and tangible structural barriers to engagement along the HIV care continuum, such as unstable or low-quality housing or homelessness and difficulty accessing high-quality HIV services $[8,9]$. Social-level barriers include complex stigma and social isolation $[9,10]$. At the individual level of influence, primary barriers include medical distrust and substance and mental health concerns $[11,12]$. Because much of the research on PLWH is conducted in HIV care settings, those poorly engaged along the HIV care continuum are understudied compared to their better-engaged peers [13-16]. The present study focuses on a subpopulation of PLWH that is both at high-risk for poor HIV outcomes and also understudied: low-SES AABL PLWH who are not engaged in HIV care at recommended levels and not virally suppressed.

Substance use, risk for hazardous substance use, and diagnosed substance use disorders are highly prevalent among PLWH (40-75\%) [17-19]. One national study found nearly half of PLWH met the criteria for a substance use disorder (48\%) [14]. In fact, HIV and substance use are considered twin or overlapping epidemics [20,21]. Substance use contributes to the transmission of HIV directly, through injection drug use, and indirectly, via pathways such as the disinhibition of sexual behavior for some and by impeding access to HIV care [22, 23]. Further, known risk factors for substance use and substance use disorders are common among PLWH. These include poverty, unemployment, unstable housing and homelessness, incarceration, chronic pain, adverse childhood experiences, continuous traumatic stress, substance-use related stigma, and difficulties accessing high-quality substance use and mental health treatment and harm reduction services [20,24]. Although rates of substance use are not higher among AABL PLWH compared to White PLWH [20, 25], AABL PLWH do commonly experience greater harms associated with substance use, including complex internalized stigma and challenges to engagement along the HIV care continuum as a result of substance use [25-27].

Alcohol, cannabis, cocaine (both powder and crack cocaine), and amphetamines are among the most commonly used substances among PLWH [14, 15, 18, 20]. Among PLWH recruited from HIV clinics, where most patients are taking ART, rates of current alcohol use, including hazardous alcohol use, are substantial. The majority have used alcohol in their lifetimes and approximately a quarter exhibit alcohol use at hazardous levels [14, 18, 20, 28, 29]. Approximately $25-50 \%$ use marijuana, an estimated $10 \%$ report amphetamine use, and $10 \%$ report crack cocaine use [20]. Lifetime injection drug use among PLWH in these settings ranges from 2 to $5 \%$, and the prevalence of current injection drug use is very low $(<1 \%)[15,20,30,31]$. Moreover, very high rates of tobacco use are found among
PLWH $(40-70 \%)[16,18,32]$. Similar to research in the general population [33], studies of substance use among PLWH usually focus on single substances. However, there is a modest literature on polysubstance use among PLWH. In a recent study of adolescents and young adults, almost one-third evidenced polysubstance use and polysubstance users experienced more mental health symptoms compared to those with minimal illicit drug use [30]. In studies of adults living with HIV, the prevalence of polysubstance use ranges from 7 to $28 \%[14,15,20,31]$.

Of concern, substance use and related substance use disorders stand out as major barriers to ART adherence, ART persistence, and sustained viral suppression [18, 34], with only cannabis found to have no adverse effects on ART adherence [15]. Moreover, substance use reduces healthrelated quality of life among PLWH [35]. Substance use is related to accelerated HIV disease progression through poor adherence to HIV care appointments and to ART and through the direct adverse effects of the substance on health $[17,18,36]$. Altice and colleagues [17] found that PLWH who use drugs have greater morbidity and earlier mortality compared with their same-aged PLWH peers who do not use drugs. Persons with injection drug use histories are less likely to receive ART and tend to have less cumulative time on ART [19]. Yet, opioid substitution therapy can ameliorate some of the risks associated with injection drug use [17]. Overall, polysubstance use is a stronger predictor of poor HIV health outcomes than the use of single substances [15, 30, 31].

Substance use is commonly co-morbid with mental health symptoms, and these psychiatric comorbidities complicate HIV treatment engagement $[17,18]$. In a study conducted in a large, private HIV medical care program, $25 \%$ of patients had received a psychiatric diagnosis ( $81 \%$ had major depression, $17 \%$ had panic disorder, and $14 \%$ had bipolar disorder); $26 \%$ had been diagnosed with a substance use disorder; and $12 \%$ of patients had received both psychiatric and substance diagnoses [37]. Moreover, the highest risk of death was found among patients with dual psychiatric and substance use diagnoses who had no psychiatric treatment visits and no substance treatment [37]. Moreover, trauma exposure is high among PLWH and up to $40 \%$ have symptoms of posttraumatic stress disorder (PTSD) [38]. Depressive symptoms commonly co-occur with PTSD among PLWH [39], as do substance use disorders [38]. Evidence that different substances may affect engagement along the HIV care continuum differently highlights the need for studies of patterns of substance use, including polysubstance use [31, 40].

Thus, substance use, including polysubstance use, and mental health symptoms and diagnoses are prevalent among PLWH, including AABL PLWH, complicate HIV management, and contribute to morbidity and mortality. Yet, the majority of the studies to date focus primarily 
on PLWH who are well-engaged in HIV care and highly adherent to ART. The present study seeks to advance the literature on substance use, including polysubstance use, and its relationship to critical risk factors by focusing on AABL PLWH from low-SES backgrounds who were not well-engaged along the HIV care continuum. We use latent class analysis (LCA) to explore patterns of polysubstance use and their relation to risk factors prevalent in this population; namely, financial hardship, homelessness, incarceration, and mental health symptoms. LCA [41] is a statistical method that uncovers latent subgroups, or "classes," defined by distinct response patterns on multiple relevant risk factors. LCA is well suited to examining polysubstance use because it can assess specific combinations of multiple behaviors simultaneously.

\section{Methods}

\section{Summary of the Present Study}

The present study takes a cross-sectional and quantitative approach. It draws upon data from a structured baseline assessment battery conducted between April 2017 and November 2019 as part of a larger intervention optimization trial using the multiphase optimization strategy (MOST) [42]. Conducted in New York City (NYC), the larger study focused on AABL PLWH from low-SES backgrounds who were poorly engaged along the HIV care continuum-specifically, those who did not engage in HIV care at recommended levels and who evidenced both poor adherence to ART and detectable HIV viral load. The study was approved by the Institutional Review Board at the New York University Grossman School of Medicine. In-person study activities took place in confidential offices at a project field site in lower Manhattan in NYC. Participants gave signed informed consent for study activities.

\section{Eligibility Criteria}

The larger study's inclusion criteria were: (1) age 18-65 years; (2) AABL race/ethnicity; (3) HIV diagnosed for $>6$ months (HIV status medically confirmed); (4) suboptimal engagement in HIV care [operationalized as less than one visit in every four month period in the past year (two of them at least 90 days apart), or $>2$ missed visits without prior cancellation in the past year] [43]; (5) ART adherence less than $50 \%$ in the past 6 weeks (by self-report) and detectable HIV viral load based on laboratory report; (6) residence in the NYC metropolitan area; (7) not planning to leave the NYC metropolitan area in the next year; and (8) able to conduct research activities in English or Spanish.

\section{Recruitment into the Larger Study}

Recruitment of PLWH with poor engagement along the HIV care continuum is challenging [13]. Participants were recruited using a hybrid community-based sampling strategy comprised of peer-to-peer recruitment, direct recruitment by study staff members in HIV service, HIV housing, and other community-based organizations, and advertisements placed in a local free newspaper. Peer-to-peer recruitment was tracked with a coupon system that linked the recruiter to the recruit, and recruiters received modest compensation for recruitment (\$15/recruit). Most enrolled participants were recruited by peers $(75 \%) ; 9 \%$ were recruited through newspaper ads, and $16 \%$ through other means.

\section{Procedures}

\section{First-Stage Screening}

Potential participants contacted the study directly, generally by phone, and usually with a coded recruitment coupon provided to them by a recruiter. Verbal informed consent was elicited following an IRB-approved script. Participants then engaged in a brief structured screening interview $(\sim 10 \mathrm{~min})$ administered by a staff member to determine preliminary eligibility. No compensation was provided for this brief phone screening.

\section{Second-Stage Screening (in Person)}

Participants found preliminarily eligible in the first screening interview provided signed informed consent for the second step in the screening process. Then, medical documentation to confirm HIV status was reviewed. Next, participants were escorted by a study staff member to a local commercial laboratory for HIV viral load testing. Participants were instructed on peer recruitment procedures and received recruitment coupons at this time. They were compensated $\$ 15$ for the screening interview and $\$ 15$ for completing the blood specimen draw, along with funds for local round-trip public transportation.

\section{Enrollment and Baseline Assessment}

HIV viral load results were typically available from the laboratory within 1-4 days. Those found eligible for the larger study at this stage based on meeting the HIV viral load criterion were contacted and invited to enroll in the study. Then, after providing signed informed consent for enrollment, participants completed a structured baseline assessment battery 
lasting 60-90 min. The baseline assessment was conducted on the REDCap platform and was comprised of computerassisted personal interviewing (CAPI) and audio, computerassisted self-interviewing (ACASI) formats. Individuals were compensated $\$ 25$ for the baseline assessment, along with funds for round-trip local transportation.

\section{Measures}

The structured instruments that made up the baseline assessment battery have been found reliable and valid with similar populations in high-risk contexts [44]. Cronbach's $\alpha$ for scales is reported where appropriate.

\section{Socio-Demographic and Background Characteristics, Risk Factors, and HIV-Related Health Indices}

We assessed age, race/ethnicity, sex, sexual and/or gender minority status (yes/no), incarceration history [never, in the past but not recent (past 6 months), or recent (past 6 months)], homelessness history (currently homeless, homeless in the past year but not currently, homeless in the lifetime but not in the past year, or never homeless), education level (high school diploma or equivalent or higher; yes/no), currently employed (yes/no), and the frequency of running out of funds for necessities such as rent, utilities, or food in the past 12 months, an indicator of extreme financial hardship (never, daily, weekly, monthly, occasionally). We used a version of the HIV Cost and Services Utilization Study instrument to assess years since first HIV diagnosis, whether ever took ART (yes/no), the longest period of sustained ART since diagnosis, in months, the number of times stopped and started ART, whether took ART in the six weeks before enrollment, and if not, the last time ART was taken, in months [45]. HIV viral load was coded on a $\log _{10}$ scale.

\section{Substance Use}

Substance use patterns were assessed by the World Health Organization Alcohol, Smoking and Substance Involvement Screening Test (WHO ASSIST) [46]. The ASSIST questionnaire is designed to identify people who are using substances in a hazardous way that may be creating harms. We assessed the following domains cross ten substances (tobacco products, alcohol, cannabis, cocaine, amphetamine-type stimulants, sedatives and sleeping pills, hallucinogens, inhalants, opioids, and 'other' drugs): lifetime use, frequency of recent use (past three months), and the frequency of experiencing indicators of hazardous use; namely, a strong desire or urge to use; health, social, legal or financial problems related to substance use; interference with role responsibilities related to use; whether anyone else has ever expressed concern about the participant's use of each substance; and whether the participant has ever tried to cut down or stop use of a substance and failed in that attempt. The WHO ASSIST provides algorithms for a risk score for each substance: lower risk (occasional or non-problematic use), moderate risk (more regular use) or high risk (frequent hazardous or problematic use). We created a "risk score" capturing moderate-to-high risk use (yes/no) for the more prevalent substances in this sample: tobacco, alcohol, cannabis, cocaine, methamphetamine, prescription opioids, and street opioids. The remaining substances were combined into a variable capturing moderate-to-high risk associated with other substances. We also calculated moderate-to-high-risk of polysubstance use (that is, likely moderate-to-high risk of two or more substances excluding tobacco and alcohol). Injection drug use history was assessed and coded as injection drug use lifetime, but not in the past 3 months (yes/no), injection drug use in the past three months (yes/no), and never injected drugs. We assessed engagement in any substance use treatment in the past (yes/no) as an indicator of a history of past serious, hazardous, and/or heavy substance use (e.g., outpatient drug treatment, medication for opioid use disorder such as a methadone maintenance treatment program or buprenorphine, 12-step or self-help meetings like AA or NA, and inpatient treatment).

\section{Mental Health Symptoms (Depression, Anxiety, PTSD, and Mental Health Quality of Life)}

The Patient Health Questionnaire depression module (PHQ9) is a well-validated nine-item self-administered instrument used for detecting depression and assessing the severity of depression over the prior two weeks. Higher PHQ-9 scores are associated with decreased functional status and increased symptom-related difficulties, sick days, and healthcare utilization [47]. Items such as "feeling little interest or pleasure in doing things" and "feeling down, depressed, or hopeless" were assessed on a 4-point, Likert-type scale (not at all, several days, more than half the days, nearly every day). Items were summed and scores ranged from 0 to 27 (Cronbach's $\alpha$ in this sample $=0.89$ ). The Generalized Anxiety Disorder scale (GAD-7) was used to assess symptoms of anxiety. This is a well-validated 7-item instrument that focuses on anxiety in the past two weeks and includes how often respondents have been bothered by issues such as "feeling nervous, anxious or on edge" or "not being able to stop or control worrying," scored on a 4-point, Likert-type scale (not at all, several days, more than half the days, nearly every day). Items were summed and scores ranged from 0 to 21 (Cronbach's $\alpha$ in this sample $=0.90$ ) [48]. We used the Primary Care PTSD Screen to assess symptoms of PTSD. This is a 4-item inventory assessing items such as "felt numb or detached from others, activities, or your surroundings" and 
"have had nightmares about (a trauma) or thought about it when you did not want to" on a yes/no scale. A score of 3 or more is coded as "likely PTSD" [49]. We assessed quality of life related to mental health using the 12-item Mental Health Component of the SF-12, a reliable and validated measure (Cronbach's $\alpha$ in this study $=0.79)$ [50]. We calculated a mental health composite $\mathrm{T}$ score, a weighted linear composite based on responses to all items of the SF-12 and weights presented by Ware and colleagues [50]. Mental health composite $\mathrm{T}$ scores could range from 5.89 (worst mental health) to 72.28 (best mental health). The normative mean for the mental health composite in the general U.S. population was $50[50]$.

\section{Data Analyses}

Latent class indicators included the WHO ASSIST ordered risk category (low, moderate, high) for twelve substances, history of injection drug use (never, lifetime but not past three months, past three months), and history of medication for opioid use disorder (No/Yes). Latent class models with one through eight classes were estimated and compared, and a single model was selected. Information criteria (e.g., Akaike Information Criterion and Bayesian Information Criterion), which compare relative fit of competing models with penalties for complexity, entropy (the degree to which only one latent class is highly probable for each individual), and the Vuong-Lo-Mendell-Rubin (VLMR) likelihood ratio test were all considered in model comparison. Once a latent class solution was identified, the model was extended to include mental health variables as distal outcomes using the $\mathrm{BCH}$ approach [51]. The $p$ values for pairwise comparisons of latent classes were adjusted for multiple comparisons using the Holm method [52]. Mental health variables included the mental health composite of the SF-12, the PHQ-9 depression total score, the GAD-7 anxiety total score, and whether criteria were met for likely PTSD on the screening instrument. Mental health variables were regressed on latent class membership. Moderation of associations between substance use latent class and race/ethnicity were explored by regressing outcomes variables on substance use latent class (most likely class), race/ethnicity, and their interaction in generalized linear models. All latent variable models were fit using Mplus [53]; other analyses were conducted using the R statistical computing environment [54].

\section{Results}

\section{Description of the Sample}

The sociodemographic and background characteristics of the sample are presented in Table 1. Participants were
47 years old on average ( $\mathrm{SD}=11$ years) with a range from 19 to 65 years. Approximately two-thirds (69\%) were African American or Black and the remainder were Latino or Hispanic. The majority (70\%) were cisgender men. A total of $58 \%$ of participants were sexual minorities (that is, lesbian, gay, queer, or otherwise not heterosexual in orientation, and/or a man who has sex with men or a woman who has sex with women) and $8 \%$ were transgender. Approximately half $(47 \%)$ had been incarcerated previously, but not in the past 6 months, and $10 \%$ had been incarcerated in the past 6 months. Almost all (90\%) had been homeless in the past and $41 \%$ were currently homeless. The majority $(70 \%)$ had a high school diploma, the equivalent or higher but employment was uncommon (8\%). A substantial proportion lacked funds for basic necessities in the past year at least monthly (43\%) or occasionally (38\%). Participants had been diagnosed with HIV 18 years ago on average ( $\mathrm{SD}=9$ years). The average HIV viral load at enrollment on the $\log _{10}$ scale was $4(\mathrm{SD}=1)$. Almost all $(98 \%)$ had taken ART in the past, and among those who had taken ART, the mean longest interval of sustained ART was 35 months ( $\mathrm{SD}=47$ months). The average number of times participants had started ART was 12 times ( $\mathrm{SD}=24$ times). Less than half $(44 \%)$ had taken $\mathrm{ART}$ at all in the prior six-week period. Among those who had not taken ART in the past 6 weeks, the last time they had taken ART was 8 months ago, on average ( $\mathrm{SD}=26$ months).

Prevalence rates of substance use and mental health patterns are presented in Table 2 . The most commonly used substances in the past three months were tobacco (79\%), alcohol (64\%), cannabis products (58\%), and cocaine (57\%). A total of $46 \%$ used two or more substances in the past three months, excluding tobacco and alcohol. Injection drug use was infrequent, with $13 \%$ having injected in their lifetimes but not recently, and an additional 9\% having injected in the past three months. The WHO ASSIST risk scores followed a similar pattern, where moderate-to-high-risk use scores were common for tobacco (83\%), alcohol (46\%), cannabis (63\%), and cocaine (56\%). Half (55\%) had moderate-to-high-risk of polysubstance use. Most (72\%) had received treatment for substance use in the past, including opioid substitution therapy. Levels of mental health distress appeared moderate. PHQ-9 Depression Symptom Scores ranged from 0 to 27 (mean $=8.0, \mathrm{SD}=6.4)$. GAD Symptom Scores ranged from 0 to $14($ mean $=5.0, S D=4.4)$. Less than a third $(29 \%)$ met the criteria for likely PTSD. The SF-12 mental health composite $\mathrm{T}$ scores ranged from 14.4 to 66.5 and the mean $\mathrm{T}$ score in this sample was below average $($ mean $=44.2$, $\mathrm{SD}=11.7$ ).

\section{Substance Use Latent Classes}

Latent class models with one to eight classes were compared (see Table 3). The Akaike Information Criterion (AIC) 
Table 1 Sociodemographic and background characteristics and risk factors $(N=512)$
$N[\%$, mean $(\mathrm{SD})]$

Age in years at baseline

Mean (SD)

$47.0(10.7)$

Median [min, max]

$50.0[19.0,65.0]$

African American or Black (non-Hispanic)

$351(68.6 \%)$

Hispanic or Latinx

$161(31.4 \%)$

Sex assigned at birth

Female

$153(29.9 \%)$

Male

$359(70.1 \%)$

Sexual minority (lesbian, gay, queer, homosexual, non-heterosexual)

$297(58.0 \%)$

Transgender

Sexual and/or gender minority

$41(8.0 \%)$

$303(59.2 \%)$

Incarceration

Never

$211(41.2 \%)$

Past, not recent

$240(46.9 \%)$

Recent

$52(10.2 \%)$

Homelessness history

Currently homeless

$211(41.2 \%)$

Homeless in past year, not currently

$56(10.9 \%)$

Homeless lifetime, not in past year

$191(37.3 \%)$

Never homeless

$54(10.5 \%)$

Education

HS diploma or equivalent or higher

$359(70.1 \%)$

Currently employed

$42(8.2 \%)$

During the past 12 months, how often did you run out of money for necessities like rent, utilities, or food?

Never

$92(18.0 \%)$

Daily

Weekly

$32(6.2 \%)$

Monthly

$66(12.9 \%)$

Occasionally

$122(23.8 \%)$

$194(37.9 \%)$

Years since HIV diagnosis

Mean (SD)

$18.2(8.61)$

Median [min, max]

$19.0[0,30.0]$

HIV viral load $\left(\log _{10}\right)$

Mean (SD)

$3.77(1.20)$

Median [min, max]

$4.04[1.30,6.35]$

Ever took ART

$504(98.4 \%)$

If yes, longest period on ART in the past (months)

Mean (SD)

$34.5(46.8)$

Median [min, max]

$18[0,297]$

If yes, number of times started ART

Mean (SD)

$11.5(23.6)$

Median [min, max]

$5[0,300]$

If yes, took ART at all in 6 weeks before enrollment

$224(44.4 \%)$

If no (did not take ART in past 6 weeks), last time took ART (months ago)

Mean (SD)

$7.69(26.1)$

Median [min, $\max ]$
$2.03[1,292]$ 
Table 2 Substance use prevalence and patterns and mental health, $[N, \%$ or mean $(\mathrm{SD})$, median]
Any tobacco past 3 months

$403(78.7 \%)$

Any alcohol past 3 months

$326(63.7 \%)$

Any cannabis past 3 months

$299(58.4 \%)$

Any cocaine past 3 months

$293(57.2 \%)$

Any methamphetamine past 3 months

$76(14.8 \%)$

$70(13.7 \%)$

$55(10.7 \%)$

$106(20.7 \%)$

$234(45.7 \%)$

Any polysubstance use past 3 months ( $2+$ substances excluding tobacco and alcohol)

Have you ever used any drug by injection (non-medical use only)?

No, never

$402(78.5 \%)$

Yes, but not in the past 3 months

$65(12.7 \%)$

Yes, in the past 3 months

$45(8.8 \%)$

$87(17.0 \%)$

$423(82.6 \%)$

$233(45.5 \%)$

$321(62.7 \%)$

$288(56.2 \%)$

$97(18.9 \%)$

$88(17.2 \%)$

$73(14.3 \%)$

$125(24.4 \%)$

$281(54.9 \%)$

$370(72.3 \%)$

Any substance use treatment-lifetime

PHQ-9 depression symptom score

Mean (SD)

$8.00(6.37)$

Median [min, max]

$7.00[0.00,27.0]$

Generalized anxiety disorder symptom score

Mean (SD)

$4.96(4.39)$

Median [min, max]

$4.67[0.00,14.0]$

$149(29.1 \%)$

Likely PTSD

SF-12 mental health composite T-score

Mean (SD)

$44.2(11.7)$

Median [min, max]
Table 3 Latent class model selection information

\begin{tabular}{lclllll}
\hline Model & Parameters & AIC & BIC & Entropy & $\begin{array}{l}\text { VLMR-2LL } \\
\text { difference }\end{array}$ & VLMR p value \\
\hline 1 class & 27 & 8409.413 & 8523.848 & - & & - \\
2 classes & 55 & 7783.157 & 8016.265 & 0.875 & 682.256 & 0.0011 \\
3 classes & 83 & 7618.293 & 7970.074 & 0.888 & 220.864 & 0.2545 \\
4 classes & 111 & 7521.910 & 7992.364 & 0.798 & 152.383 & 0.7660 \\
5 classes & 139 & 7431.403 & 8020.530 & 0.843 & 146.507 & 0.8426 \\
6 classes & 167 & 7346.757 & 8054.557 & 0.847 & 140.343 & 0.7658 \\
7 Classes & 195 & 7292.880 & 8119.353 & 0.862 & 109.877 & 0.7652 \\
8 classes & 223 & 7289.416 & 8234.562 & 0.871 & 60.067 & 0.8461 \\
\hline
\end{tabular}

value generally favors more complex models, and so was not used in model selection. The Vuong-Lo-Mendell-Rubin likelihood ratio tests showed no support for models with more than three latent classes. The three-class model had the lowest Bayesian Information Criterion (BIC) value and 
Table 4 Three substance use classes: class prevalences and risk level probabilities

\begin{tabular}{|c|c|c|c|c|}
\hline Indicator & Risk level & $\begin{array}{l}\text { Class 1 }(n=44 ; \\
8.6 \%)\end{array}$ & $\begin{array}{l}\text { Class 2 }(n=90 \\
17.6 \%)\end{array}$ & $\begin{array}{l}\text { Class 3 } \\
(n=378 \\
73.8 \%)\end{array}$ \\
\hline \multirow[t]{3}{*}{ Tobacco } & Low & 0.075 & 0.030 & 0.220 \\
\hline & Moderate & 0.411 & 0.838 & 0.668 \\
\hline & High & 0.514 & 0.132 & 0.112 \\
\hline \multirow[t]{3}{*}{ Alcohol } & Low & 0.447 & 0.494 & 0.569 \\
\hline & Moderate & 0.176 & 0.415 & 0.298 \\
\hline & High & 0.377 & 0.091 & 0.133 \\
\hline \multirow[t]{3}{*}{ Cannabis } & Low & 0.400 & 0.134 & 0.427 \\
\hline & Moderate & 0.167 & 0.757 & 0.475 \\
\hline & High & 0.433 & 0.108 & 0.098 \\
\hline \multirow[t]{3}{*}{ Cocaine } & Low & 0.290 & 0.155 & 0.522 \\
\hline & Moderate & 0.097 & 0.700 & 0.364 \\
\hline & High & 0.613 & 0.146 & 0.113 \\
\hline \multirow[t]{3}{*}{ Methamphetamine } & Low & 0.771 & 0.452 & 0.901 \\
\hline & Moderate & 0.025 & 0.441 & 0.095 \\
\hline & High & 0.204 & 0.107 & 0.004 \\
\hline \multirow[t]{3}{*}{ Prescription stimulants } & Low & 0.850 & 0.778 & 0.980 \\
\hline & Moderate & 0.036 & 0.222 & 0.020 \\
\hline & High & 0.114 & 0.000 & 0.000 \\
\hline \multirow[t]{3}{*}{ Hallucinogens } & Low & 0.829 & 0.619 & 0.984 \\
\hline & Moderate & 0.080 & 0.381 & 0.013 \\
\hline & High & 0.091 & 0.000 & 0.003 \\
\hline \multirow[t]{3}{*}{ Inhalants } & Low & 0.843 & 0.578 & 0.974 \\
\hline & Moderate & 0.066 & 0.422 & 0.026 \\
\hline & High & 0.091 & 0.000 & 0.000 \\
\hline \multirow[t]{3}{*}{ Prescription opioids } & Low & 0.553 & 0.494 & 0.940 \\
\hline & Moderate & 0.197 & 0.506 & 0.052 \\
\hline & High & 0.250 & 0.000 & 0.008 \\
\hline \multirow[t]{3}{*}{ Street opioids } & Low & 0.502 & 0.441 & 0.999 \\
\hline & Moderate & 0.085 & 0.506 & 0.001 \\
\hline & High & 0.412 & 0.054 & 0.000 \\
\hline \multirow[t]{3}{*}{ Sedatives } & Low & 0.675 & 0.524 & 0.959 \\
\hline & Moderate & 0.032 & 0.476 & 0.036 \\
\hline & High & 0.294 & 0.000 & 0.005 \\
\hline \multirow[t]{3}{*}{ Other drugs } & Low & 0.909 & 0.954 & 0.990 \\
\hline & Moderate & 0.000 & 0.046 & 0.008 \\
\hline & High & 0.091 & 0.000 & 0.003 \\
\hline \multirow{2}{*}{$\begin{array}{l}\text { History of medication for opioid } \\
\text { use disorder }\end{array}$} & No & 0.277 & 0.580 & 0.954 \\
\hline & Yes & 0.723 & 0.420 & 0.046 \\
\hline \multirow[t]{3}{*}{ History of injection drug use } & Never & 0.389 & 0.432 & 0.916 \\
\hline & Past only & 0.308 & 0.351 & 0.052 \\
\hline & Recent & 0.303 & 0.218 & 0.032 \\
\hline
\end{tabular}

The indicator level with the highest probability within each latent class is shown in bold font was selected as the best solution. Parameter estimates for the three-class model are presented in Table 4.

The smallest class, Class 1 , describes approximately $9 \%$ of the sample. This class is characterized by high-risk use of tobacco, cannabis, and cocaine, and a history of medication for opioid use disorder. Although members of this class were most likely to report low-risk alcohol use $(44.7 \%)$, they also had a $37.7 \%$ probability of reporting high-risk alcohol use. Similarly, although those in Class 1 were most likely to report low-risk use of street opioids 
$(50.2 \%)$, they also had a $41.2 \%$ probability of reporting high-risk use. Finally, although the plurality of this class endorsed "never" when asked about their history of injection drug use, more than $60 \%$ had used drugs by injection either in the past or recently. We refer to this as the high polysubstance use/high-risk class.

Class 2, which describes approximately $18 \%$ of the sample, is characterized by moderate-risk use of tobacco, cannabis, cocaine, prescription opioids, and street opioids, and low- to moderate-risk use of alcohol, methamphetamines, hallucinogens, and inhalants. Members of this class used a wide variety of substances without reporting high-risk use of any. Although they were most likely to report no history of medication for opioid use disorder, a substantial minority of $42 \%$ did have this history. The pattern of responses to the question about a history of injection drug use was similar to that of Class 1 , with a slightly higher proportion of respondents reporting no history. We refer to this as the polysubstance use/moderate risk class.

Class 3 is the largest, describing approximately $74 \%$ of the sample. This class is characterized by moderate-risk use of tobacco and cannabis, low- to moderate-risk use of cocaine, low-risk use of all other substances, and a very low probability of a history of either medication for opioid use disorder or injection drug use. We refer to this as the moderate polysubstance use/moderate-to-low-risk class.

\section{Association Between Substance Use Latent Classes and Risk Factors}

Table 5 shows associations between substance use latent class membership and mental health variables. On the SF-12 mental health composite, the small, higher risk class (Class 1) had poorer mental health than both Class $2\left[X^{2}(1)=8.7\right.$; $\mathrm{p}=0.018]$ and Class $3\left[\mathrm{X}^{2}(1)=21.3 ; \mathrm{p}<0.001\right]$. Also, on the PHQ-9 depression severity score, Class 1 had greater symptoms of depression than both Class $2\left[X^{2}(1)=10.0\right.$; $\mathrm{p}=0.014]$ and Class $3\left[\mathrm{X}^{2}(1)=14.0 ; \mathrm{p}<0.001\right]$. On the GAD-7 anxiety total score, Class 1 members were more anxious than members of both Class $2\left[\mathrm{X}^{2}(1)=16.3 ; \mathrm{p}<0.001\right]$ and Class $3\left[\mathrm{X}^{2}(1)=16.3 ; \mathrm{p}<0.001\right]$. Finally, a positive result on the PTSD screener was more likely in Class 1 $\left[\mathrm{X}^{2}(1)=12.0 ; \mathrm{p}=0.008\right]$ and Class $2\left[\mathrm{X}^{2}(1)=8.8 ; \mathrm{p}=0.018\right]$ when each was compared with Class 3 . Current homelessness was more likely in Class $1\left[\mathrm{X}^{2}(1)=6.2 ; \mathrm{p}=0.026\right]$ and Class $2\left[\mathrm{X}^{2}(1)=7.1 ; \mathrm{p}=0.024\right]$ when compared with Class 3. After adjustment for multiple comparisons, there were no significant differences among substance use latent classes on either financial hardship or recent incarceration.
Table 5 Differences among substance use latent classes on risk factors

\begin{tabular}{|c|c|c|c|c|c|}
\hline & Mean & SE & Comparison & Chi-square & $\mathrm{p}$ value* \\
\hline \multicolumn{6}{|c|}{ SF-12 MCS } \\
\hline Class 1 & 36.567 & 1.770 & Overall test & 21.813 & $<.001$ \\
\hline Class 2 & 43.441 & 1.410 & 1 vs 2 & 8.662 & 0.018 \\
\hline \multirow[t]{2}{*}{ Class 3} & 45.322 & 0.607 & 1 vs 3 & 21.303 & $<.001$ \\
\hline & & & 2 vs 3 & 1.424 & 0.932 \\
\hline \multicolumn{6}{|c|}{ PHQ-9 depression } \\
\hline Class 1 & 12.601 & 1.302 & Overall test & 13.946 & 0.001 \\
\hline Class 2 & 7.743 & 0.728 & 1 vs 2 & 9.978 & 0.014 \\
\hline \multirow[t]{2}{*}{ Class 3} & 7.530 & 0.323 & 1 vs 3 & 13.945 & $<.001$ \\
\hline & & & 2 vs 3 & 0.068 & 0.932 \\
\hline \multicolumn{6}{|c|}{ GAD-7 anxiety } \\
\hline Class 1 & 8.148 & 0.797 & Overall test & 17.781 & $<.001$ \\
\hline Class 2 & 4.326 & 0.455 & 1 vs 2 & 16.332 & $<.001$ \\
\hline \multirow[t]{2}{*}{ Class 3} & 4.754 & 0.234 & 1 vs 3 & 16.266 & $<.001$ \\
\hline & & & 2 vs 3 & 0.664 & 0.932 \\
\hline \multicolumn{6}{|l|}{ PTSD } \\
\hline Class 1 & 0.542 & 0.086 & Overall test & 19.606 & $<.001$ \\
\hline Class 2 & 0.420 & 0.058 & 1 vs 2 & 1.312 & 0.932 \\
\hline \multirow[t]{2}{*}{ Class 3} & 0.231 & 0.023 & 1 vs 3 & 12.000 & 0.008 \\
\hline & & & 2 vs 3 & 8.843 & 0.018 \\
\hline \multicolumn{6}{|c|}{ Financial hardship } \\
\hline Class 1 & 0.601 & 0.086 & Overall test & 8.610 & 0.014 \\
\hline Class 2 & 0.528 & 0.059 & 1 vs 2 & 0.463 & 0.496 \\
\hline \multirow[t]{2}{*}{ Class 3} & 0.393 & 0.026 & 1 vs 3 & 5.236 & 0.066 \\
\hline & & & 2 vs 3 & 4.154 & 0.084 \\
\hline \multicolumn{6}{|c|}{ Current homelessness } \\
\hline Class 1 & 0.586 & 0.085 & Overall test & 12.180 & 0.002 \\
\hline Class 2 & 0.536 & 0.058 & 1 vs 2 & 0.219 & 0.640 \\
\hline \multirow[t]{2}{*}{ Class 3} & 0.362 & 0.026 & 1 vs 3 & 6.204 & 0.026 \\
\hline & & & 2 vs 3 & 7.056 & 0.024 \\
\hline \multicolumn{6}{|c|}{ Recent incarceration } \\
\hline Class 1 & 0.180 & 0.065 & Overall test & 4.600 & 0.100 \\
\hline Class 2 & 0.158 & 0.043 & 1 vs 2 & 0.072 & 0.788 \\
\hline \multirow[t]{2}{*}{ Class 3} & 0.082 & 0.015 & 1 vs 3 & 2.109 & 0.306 \\
\hline & & & 2 vs 3 & 2.676 & 0.306 \\
\hline
\end{tabular}

*Pairwise comparison p values were adjusted for multiple comparisons using the Holm [52] method

\section{Moderation of Associations By Race/Ethnicity}

An interaction effect was found between substance use latent class and race/ethnicity for GAD-7 anxiety scores $[F(2,493)=3.5, p=0.031]$. Analysis of simple main effects within each racial/ethnic group showed differences in anxiety scores among Latinos between Class 1 and Class 2 [mean difference $=6.3 ; \mathrm{SE}=1.4 ; \mathrm{t}(493)=-4.5, \mathrm{p}<0.001$ ] and between Class 1 and Class 3 [mean difference $=4.7$; $\mathrm{SE}=1.2 ; \mathrm{t}(493)=-3.8, \mathrm{p}<0.001]$ were larger and significant, but smaller and not significant among African 
American/Black (non-Latino) participants (data not included in tables).

\section{Discussion}

Co-occurring polysubstance use and mental health symptoms have the potential to place PLWH at grave risk for adverse physical health outcomes and poor quality of life, as described above. But polysubstance use has received relatively little attention in the literature compared to studies of single substances. The present cross-sectional quantitative study sought to add depth and nuance to our understanding of the relationships among patterns of polysubstance use and their associations with a set of risk factors in an important but under-studied subpopulation of PLWH: AABL individuals from low-SES backgrounds who are poorly engaged along the HIV care continuum. Overall, we found the prevalence of recent substance use across a range of substances is higher in this subpopulation of PLWH than in past published reports that focus mainly on those well-engaged in HIV care. Moreover, whereas rates of polysubstance use are certainly substantial in past studies $(7-28 \%)[15,20,31]$, over half of those in the present study exhibit polysubstance use at moderate- to high-risk levels, even when excluding alcohol and tobacco. We found LCA was useful in drawing out patterns of substance use at varying levels of risk across a range of substances, along with their relation to risk factors and co-occurring mental health symptoms. Findings highlight that small subgroups of AABL PLWH experience multiple, serious, complex challenges, and that the majority in this sample use substances, including multiple substances, at levels ranging from moderate- to low-risk use.

Most participants in the present study (approximately 75\%) are classified in a group described as moderate polysubstance use/low-to-moderate substance use risk (Class $3)$. Class 3 differs from the other two classes in that injection drug use is absent, risk levels for alcohol, cannabis, or cocaine are lower than in the other classes, although not absent, and risk levels for opioids, stimulants other than cocaine, and other drugs are low. We compared these findings to past research. Parsons and colleagues [31] examined latent classes of substance use among PLWH in a clinical setting (69\% African American/Black, 17\% Latino) and found five distinct patterns: a no-use class (16\%), exclusive alcohol use (19\%); alcohol and cannabis (15\%); alcohol and cocaine/crack (26\%); and polysubstance use (24\%). The polysubstance use class in the Parsons study was comprised of those with a high rate of alcohol, cannabis, and cocaine/ crack use, as well as weekly use of opioids and other drugs. Moreover, the latter two classes reported the greatest impairment from substance use; specifically, more missed ART doses than those in the other classes [31]. Yet, our analysis found no classes where the risk for substance use was absent or very low. This does not necessarily mean there were no such individuals in the sample. It is possible a low-risk latent class would have emerged in a solution with more latent classes. However, it would likely be very small, because the presence of a large fourth latent class would likely have resulted in fit statistics that pointed toward a four-class solution rather than a three-class solution. In the present study, some types of polysubstance use are common even in the lowest-risk class. Substance use risk ranges from low to moderate, suggesting that not all in Class 3 use illicit drugs at hazardous levels. On the other hand, Class 3 was associated with mental health quality of life below the national norm, and rates of mental health symptoms were substantial.

The second-largest class describes approximately $18 \%$ of the sample. The polysubstance use/moderate-risk class has high probabilities of moderate-risk across multiple substances, including tobacco, alcohol, cannabis, stimulants, and opioids. A history of medication for opioid use disorder is common in Class 2, though not as common as for Class 1 , as we describe below. In our examination of relationships among latent classes and risk factors and mental health symptoms, Classes 2 and 3 are similar to each other in that mental health quality of life scores are below the national norm, and symptoms of depression, anxiety, and the prevalence of likely PTSD are substantial. Class 1, the high polysubstance use/high-risk substance use class, although small (9\% of participants), has substantially more polysubstance use across more substances and higher risk for hazardous levels of use, and well as a pattern of significantly lower mental health quality of life and higher rates of depression and anxiety symptoms and likely PTSD compared to Classes 1 and 2. Thus, across all three classes, polysubstance use and mental health concerns are strongly associated in this population of AABL PLWH, similar to past studies, and those in the highest-risk substance use class with the most complex patterns of serious polysubstance use have the greatest mental health concerns [55]. Regarding the risk factors studied, current homelessness is more likely in Classes 1 and 2 compared with Class 3, but financial hardship or recent incarceration do not differ among substance use latent classes. Recent incarceration was relatively uncommon in this sample, and it is possible that our measure of financial hardship lacked precision, as it examined only one aspect of extreme poverty. Homelessness has been documented as a cause of, contributing factor to, and/or consequence of substance use among PLWH [56, 57], and a fundamental cause of HIV disease progression [57]. It is also a potentially modifiable risk factor, and "housing first" interventions have great potential to serve as a foundation for improved HIV health and better management of substance use and mental health symptoms [58, 59]. 
As a cross-sectional study, temporal patterns or causal relationships are not examined. Yet, the literature sheds some light on these issues. In fact, as Marel et al. [60] note, relatively little is known about factors that may lead to the development of a substance use disorder across a range of drug classes. One recent national longitudinal study points to demographic and mental health-related factors as contributors to polysubstance use problems [61]. These included being male, younger age, never married, sexual minority identity, nicotine dependence, and anxiety, mood and personality disorders, which were associated with an increased odds of developing multiple substance use disorders and having three-year persistence of multiple substance use disorders. Moreover, this study found the majority with multiple past-year substance use disorders had a lifetime personality disorder and did not use substance abuse treatment or otherwise seek help [61]. Although the present study does not assess personality disorders, the co-occurrence of serious polysubstance use and high risk for substance problems and mental health distress are consistent with this national study. Moreover, pre-existing mental health disorders are significantly associated with an increased risk of developing substance use disorders for alcohol, cannabis, and stimulants [60]. In a study in the US Veteran's Administration, veterans with higher levels of polysubstance use disorders were more likely to be Black and homeless, were more likely to have schizophrenia, bipolar disorder, major depressive disorder, and personality disorders [33]. In another longitudinal study in the general population, substance use disorder persistence rates were consistently higher among polysubstance users, although patterns were mixed in relation to gender and race/ ethnicity [62]. Among men, Latino individuals with substance use disorders were less likely to persist than Whites. Black men with an alcohol or drug use disorder were less likely to persist than Whites, but Black men with a polysubstance use disorder were more likely to persist than Latino individuals [62]. Further, Marel et al. [60] found a relatively quick transition from substance use to substance use disorders. They emphasize a narrow window of time available to intervene, underscoring the urgency of early identification of mental health conditions and the timely provision of appropriate evidence-based interventions, which could potentially prevent the development of secondary substance use disorders [60]. Taken together, these studies highlight a number of risk factors for polysubstance use, including sociodemographic and background characteristics and mental health disorders, unique features associated with polysubstance use, the potential for persistence of substance use problems, and the challenges of effective treatment.

The fact that co-occurring polysubstance use and mental health symptoms are so common across the three classes suggests gaps in screening and treatment. There is a substantial literature on the complexities of treating co-morbid symptoms among those in the general underlying population. The literature highlights a number of successes, such as a set of effective, evidence-based treatments for substance use disorders [63, 64], as well as gaps. With regard to the latter, one major gap is that programs do not always provide a diagnosis of both substance use and mental health concerns or integrated care, in part because mental health and substance use treatment services are funded separately [63, 65-67]. Further, the effectiveness for treatment of polysubstance use is poor: Patients have serious individual- and structural-level challenges accessing treatment; drop-out rates from treatment are high (perhaps suggesting patients are not getting their needs met in treatment settings); and there is a serious lack of research on AABL and low-SES populations [63, 65-67].

PLWH with co-occurring disorders are referred to as "triply diagnosed" [68]. The HIV/AIDS Treatment Adherence, Health Outcomes and Cost Study, called "the Cost Study", examined the issue of triple diagnosis and optimal treatment models for this subpopulation of PLWH [69]. The literature suggests PLWH may be at somewhat of an advantage compared to their peers in the underlying general population because most are already linked to care settings that prioritize integrated care [70]. Yet, the Cost study found that only $33 \%$ of study participants with co-occurring disorders received concurrent treatment for substance use problems and mental health disorders, despite meeting diagnostic criteria for both: $26 \%$ received only mental health services, $15 \%$ received only substance abuse services, and $26 \%$ received no services, with AABL PLWH less likely to receive treatment for co-occurring disorders than White PLWH [71], similar to other studies that highlight low rates of service use for co-occurring disorders [72]. Despite generally being linked to comprehensive primary care settings related to the HIV diagnosis, PLWH appear to have similar barriers to engagement in effective treatment for co-occurring disorders as their peers not living with HIV [72].

Calsyn and colleagues [73] outline strategies for engaging and retaining individuals with triple diagnoses in both treatment and research. They recommend comprehensive services that are provided on a 24-h basis in a flexible and culturally competent manner and a team approach to treatment [73]. The literature on dually diagnosed persons who are not living with HIV also sheds light on characteristics of optimal treatment characteristics for co-occurring disorders. These include adopting a low-stress approach, cognitive-behavioral therapy, residential rehabilitation, contingency management, relapse prevention, engaging the social network, and non-abstinence-based supportive housing [17, 74-77]. Harm reduction and approaches to enhance motivation by supporting autonomy including motivational interviewing have promise [74, 78]. Byrd et al. [79] reported on a Patientcentered HIV Care Model that integrated community-based 
pharmacists with medical providers and required sharing of patient clinical information and collaborative therapy-related action planning. This approach improved retention and HIV viral suppression among those triply diagnosed [79].

Patterns of findings from Class 1, the high polysubstance use/highest risk substance use class, suggest some participants have faced long histories of polysubstance use risk and treatment experiences, including medication for opioid use disorder. There is a consensus that opioid substitution therapies; namely, buprenorphine and methadone, are associated with reductions in overdose and serious opioid-related acute care use compared with other treatments including among PLWH $[80,81]$. Moreover, both buprenorphine and methadone improve HIV viral suppression, adherence to antiretroviral therapy, and overall mortality for PLWH with opioid use disorder, along with improved stability and quality of life, reductions in opioid use and relapse, and reductions in the transmission of HIV infections to others [82-86]. Further, extended-release naltrexone has been studied in PLWH leaving incarcerated settings and improves HIV viral suppression in that context [82].

Yet, medication for opioid use disorders tends to be under-used among PLWH [17, 87]. Barriers to the provision of medication for opioid use disorder are numerous and operate at the levels of policies (e.g., insufficient number of waivers for buprenorphine prescription, insurance restrictions), the health care system (e.g., lack of low-threshold services), specific institutions (e.g., syringe service programs not commonly co-located in HIV care settings), health care providers (e.g., barriers to obtaining buprenorphine prescribing waivers, bias toward persons who use substances, lack of training in harm reduction), and among PLWH (e.g., feeling dehumanized, fear, mistrust, competing priorities, substance use-related stigma) $[9,84,88,89]$. Harm reduction services are critical for persons who use substances, including those who use opioids but are sorely lacking [84]. In particular, the number of syringe services programs is insufficient to meet the need, restrictions on the numbers of syringes PLWH can access increases risk, and, as noted above, such services are rarely co-located in HIV care settings [84]. These barriers to medication for opioid use disorders have serious adverse consequences for AABL PLWH and impede the larger public health goal of ending the HIV epidemic.

Further, we found stimulant use (cocaine, methamphetamine) at moderate-to-high risk levels is common in this subpopulation of AABL PLWH. Clearly, stimulant use is associated with poor HIV outcomes, including among men who have sex with men living with HIV [90-93]. But, in contrast to opioids use disorders, medications for stimulant use problems are in an earlier stage of development and are not widely used [94]. This may be in part because the use of pharmacologic management of substance use in triply diagnosed patients raises challenges such as side effects and drug interactions [76]. Effective behavioral treatments for stimulant use problems are similar to those for other single drug use and polysubstance use problems described above $[17,75,76]$. However, a review by Rajasingham et al. [92] found limited evidence of highly effective pharmacotherapy or behavioral treatments for PLWH with methamphetamine dependence. Nonetheless, integrating substance use treatment services into HIV care and drawing in particular on evidence-based and harm reduction approaches, and pharmacotherapy where appropriate, is critical $[17,83,86]$. At the same time, continued research on treatment modalities as well as on implementation strategies to improve uptake of effective interventions has the potential to improve engagement along the HIV care continuum for AABL PLWH with or at-risk for moderate-to-high-risk substance use $[17,83$, 86].

We did not expect race/ethnicity to be a moderator of associations between substance use classes and risk factors. Overall, associations were comparable for African American/Black and Latino PLWH. One exception was the anxiety outcome, where differences among substance use classes were larger among Latinos. Perhaps other factors impacting anxiety among African American/Black PLWH (e.g., everyday racism) diminish the role of substance use in that group. Since the substance use class with elevated anxiety (Class 1) was relatively rare, more work is needed for more precise estimates of how the association between substance use and anxiety may be moderated by race/ethnicity.

\section{Limitations}

Because all interview questions were asked at the same time, we do not know the temporal patterns of substance use and mental health symptoms. Longitudinal research that considers transitions in patterns and severity of substance use and mental health symptoms is needed to make causal inferences. These findings are further limited by the specific measures of mental health symptoms employed, which were brief and may have lacked precision. A more comprehensive assessment of mental health symptoms along with personality disorders and other personality factors might yield additional insights into how mental health relates to substance use classes. Moreover, substance use patterns vary by geographic region in the United States. Crane et al. [95] recently examined regional variation in substance use among PLWH and found notable differences. For example, marijuana and methamphetamine are more common in the West and Northeast, and PLWH in the Northeast are more likely to use cocaine/crack than those in the West [95]. The present study was conducted in the Northeast, and thus findings may not generalize to all geographical regions in the United States. Further, participants in the present study were longterm survivors of HIV, and older, and thus findings may not 
generalize to younger and more recently diagnosed AABL PLWH, or to White PLWH.

\section{Implications}

The present study yields a number of implications for clinical practice and future research. First, results highlight the importance of the population of AABL PLWH who are poorly engaged along the HIV care continuum, who on the whole evidence co-morbid polysubstance use and mental health distress that would clearly complicate HIV management. With additional resources, clinical settings can develop outreach and engagement approaches to bring this subpopulation of AABL PLWH to HIV care settings, and/or can partner with community-based organizations to facilitate outreach efforts. The hybrid recruitment strategy used in the present study can serve as a model for such outreach efforts. Moreover, specialized services are needed in HIV care settings to successfully treat and retain patients with triple diagnoses [96]. Findings further suggest the need for ongoing screening for substance use, including polysubstance use, and mental health comorbidity, as a vital part of improving HIV care engagement, medication adherence, maintaining overall health, and strengthening secondary prevention efforts [31]. The present study suggests we cannot "end the HIV epidemic" or eliminate racial/ethnic disparities in HIV health outcomes without locating, engaging, supporting, and successfully treating AABL PLWH who have serious barriers to engaging along the HIV care continuum. Clearly, given the complexity of the problem of triple diagnosis, including the high prevalence of polysubstance use, continued research on integrated treatment and other innovative approaches is warranted.

\section{Conclusions}

LCA revealed a small number of substance use profiles for the subpopulation of AABL PLWH who are poorly engaged along the HIV care continuum-all comprising substantial polysubstance use, although only a minority at high-risk levels. Mental health symptoms are common within all three of these profiles, and the highest-risk substance use class is associated with the lowest mental health quality of life and more mental health symptoms. These comorbidities interfere with HIV care, but there are barriers to their effective treatment. Study findings will be of interest to HIV policymakers, HIV care providers, and interventionists.

Acknowledgements The study was sponsored by the National Institute on Drug Abuse [(NIDA), R01DA040480; Drs. Marya Gwadz and Linda M. Collins, MPI; and P30DA011041, Center for Drug Use and HIV Research (CDUHR), Dr. Holly Hagan, Principal Investigator] at the National Institutes of Health. We gratefully acknowledge our Program Official at NIDA, Dr. Richard Jenkins, for guidance throughout the study, as well as Dr. Shoshana Kahana at NIDA, and Bruss Del Valle and Pauline Lee in the Office for Research at the NYU Silver School of Social Work. We wish to acknowledge Dr. Gail Melkus, Mindy Belkin, William McCormack, and Mary Lou Atkinson at the NYU Meyers College of Nursing for their support of the project. Amanda Applegate provided editorial assistance. The HTH2 Collaborative Research Team members, past and present, include J. Yvette Allen, Mariam Ayvazyan, Nico Bobb, Dr. Scott Braithwaite, Adonis Brooks, Dr. Charles M. Cleland, Sabrina Cluesman, Camille Collett, Dr. Caroline Dorsen, Dan Ediriweera, Brooks Fassett, Krystel Francis, Robert M. Freeman, Gail Gonzales, Ning He, Elona Hoffeld, Theresa Hroncich, Eshika Kalam, Sarah Kaufman, Daniel Kelly, Donna Kennedy, Alexis Korman, Dr. Alix Kutnick, Dr. Noelle Leonard, Maria Levine, Belkis Martinez, Sarah Ory, Shantae Owens, Anisha Pal, Emily Pan, Danny Quinones, Amanda Ritchie, Dawa Sherpa, Elizabeth Silverman, Stephen Sorgi, Aradhana Srinagesh, Karen Torbjornsen, Catherine Tumminello, Dr. Leo Wilton, and Andrea Wade. Most importantly, we gratefully acknowledge the study participants for their vital contributions to the study.

Author Contributions CMC conceptualized the purpose and design of the study, carried out the data analysis, and wrote much of the first draft of the article. MG acquired the funding along with LMC, helped conceptualize the purpose of the study, and wrote much of the first draft the article. In addition to acquiring study funding, LMC assisted with the data analysis plan. LW is a study Co-Investigator and assisted with interpretation of findings. DS, CD, SC, BYM, ASR, and MA played leadership roles in the larger study, including planning and guiding participant recruitment and data collection, and assisted with interpretation of findings. NRL is a study Co-Investigator and assisted with interpretation of findings. All authors commented on previous versions of the manuscript and read and approved the final manuscript.

Funding The research reported in this paper was funded by the National Institute on Drug Abuse (NIDA) at the National Institutes of Health (R01DA040480, Drs. Marya Gwadz and Linda M. Collins, Co-Principal Investigators), and the NIDA-funded Center for Drug Use and HIV Research (P30DA011041; Dr. Holly Hagan, Principal Investigator).

Data Availability Data will be placed in a repository. Contact the corresponding author for details.

\section{Declarations}

Conflict of Interest The authors declare that they have no conflicts of interest.

Ethical Approval Procedures were approved by the Institutional Review Board at the New York University Grossman School of Medicine.

Informed Consent Participants gave informed consent for study activities.

\section{References}

1. Centers for Disease Control and Prevention. Understanding the HIV care continuum. National center for HIV/AIDS, viral 
hepatitis, STD, and TB prevention. Atlanta: Centers for Disease Control and Prevention; 2019.

2. Crepaz N, Dong X, Wang X, Hernandez AL, Hall HI. Racial and ethnic disparities in sustained viral suppression and transmission risk potential among persons receiving HIV care: United States, 2014. MMWR Morb Mortal Wkly Rep. 2018;64(4):113-8.

3. Thrasher AD, Earp JA, Golin CE, Zimmer CR. Discrimination, distrust, and racial/ethnic disparities in antiretroviral therapy adherence among a national sample of HIV-infected patients. J Acquir Immune Defic Syndr. 2008;49(1):84-93.

4. Siddiqi AE, Hu XH, Hall HI. Mortality among blacks or African Americans with HIV infection: United States, 2008-2012. MMWR Morb Mortal Wkly Rep. 2015;64(4):81-6.

5. Siddiqi AE, Hall HI, Hu X, Song R. Population-based estimates of life expectancy after HIV diagnosis: United States 2008 2011. J Acquir Immune Defic Syndr. 2016;72(2):230-6.

6. Dasgupta S, Oster AM, Li J, Hall HI. Disparities in consistent retention in HIV care: 11 states and the District of Columbia, 2011-2013. MMWR Morb Mortal Wkly Rep. 2016;65(4):77-82.

7. Eberhart MG, Yehia BR, Hillier A, et al. Individual and community factors associated with geographic clusters of poor HIV care retention and poor viral suppression. J Acquir Immune Defic Syndr. 2015;69(Suppl 1):S37-43.

8. Aidala AA, Wilson MG, Shubert V, et al. Housing status, medical care, and health outcomes among people living with HIV/AIDS: a systematic review. Am J Public Health. 2016;106(1):e1-23.

9. Freeman R, Gwadz M, Wilton L, et al. Understanding long-term HIV survivorship among African American/Black and Latinx persons living with HIV in the United States: a qualitative exploration through the lens of symbolic violence. Int J Equity Health. 2020;19(1):1-23.

10. Rice WS, Crockett KB, Mugavero MJ, et al. Association between internalized HIV-related stigma and HIV care visit adherence. J Acquir Immune Defic Syndr. 2017;76(5):482-7.

11. Brincks AM, Shiu-Yee K, Metsch LR, et al. Physician mistrust, medical system mistrust, and perceived discrimination: associations with HIV care engagement and viral load. AIDS Behav. 2019;23(10):2859-69.

12. Kalichman SC, Eaton L, Kalichman MO, et al. Race-based medical mistrust, medication beliefs and HIV treatment adherence: test of a mediation model in people living with HIV/AIDS. J Behav Med. 2016;39(6):1056-64.

13. Gwadz M, Cleland CM, Applegate E, et al. Behavioral intervention improves treatment outcomes among HIV-infected individuals who have delayed, declined, or discontinued antiretroviral therapy: a randomized controlled trial of a novel intervention. AIDS Behav. 2015;19(10):1801-17.

14. Hartzler B, Dombrowski JC, Crane HM, et al. Prevalence and predictors of substance use disorders among HIV care enrollees in the United States. AIDS Behav. 2017;21(4):1138-48.

15. Mimiaga MJ, Reisner SL, Grasso C, et al. Substance use among HIV-infected patients engaged in primary care in the United States: findings from the Centers for AIDS Research Network of Integrated Clinical Systems cohort. Am J Public Health. 2013;103(8):1457-67.

16. Miles DRB, Bilal U, Hutton HE, et al. Tobacco smoking, substance use, and mental health symptoms in people with HIV in an urban HIV clinic. J Health Care Poor Underserved. 2019;30(3):1083-102.

17. Altice FL, Kamarulzaman A, Soriano VV, Schechter M, Friedland GH. Treatment of medical, psychiatric, and substance-use comorbidities in people infected with HIV who use drugs. Lancet. 2010;376(9738):367-87.
18. Gonzalez A, Barinas J, O'Cleirigh C. Substance use: impact on adherence and HIV medical treatment. Curr HIV/AIDS Rep. 2011;8(4):223.

19. McGowan CC, Weinstein DD, Samenow CP, et al. Drug use and receipt of highly active antiretroviral therapy among HIV-infected persons in two U.S. clinic cohorts. PLoS ONE. 2011;6(4):e18462.

20. Pence BW, Thielman NM, Whetten K, et al. Coping strategies and patterns of alcohol and drug use among HIV-infected patients in the United States Southeast. AIDS Patient Care STDS. 2008;22(11):869-77.

21. Welch KJ. Correlates of alcohol and/or drug use among HIV-infected individuals. AIDS Patient Care STDS. 2000;14(6):317-23.

22. Klinkenberg WD, Sacks S, HIV/AIDS Treatment Adherence Health Outcomes Cost Study Group. Mental disorders and drug abuse in persons living with HIV/AIDS. AIDS Care. 2004;16(Suppl 1):S22-42.

23. Gwadz M, de Guzman R, Freeman R, et al. Exploring how substance use impedes engagement along the HIV care continuum: a qualitative study. Front Public Health. 2016;4:62.

24. Lum PJ, Bruce RD. Comorbid substance use among persons with HIV and chronic pain. In: Merlin JS, Selwyn PA, Treisman GJ, Giovanniello AG, editors. Chronic pain and HIV. Hoboken, NJ: Wiley; 2016. p. 78-96.

25. Brown MJ, Serovich JM, Laschober TC, Kimberly JA. Age and racial disparities in substance use and self-reported viral suppression among men who have sex with men with HIV. Int J STD AIDS. 2018;29(12):1174-82.

26. Earnshaw VA, Bogart LM, Dovidio JF, Williams DR. Stigma and racial/ethnic HIV disparities: moving toward resilience. Stigma Health. 2015;1:60-74.

27. Storholm ED, Bogart LM, Mutchler MG, et al. Antiretroviral adherence trajectories among black Americans living with HIV. AIDS Behav. 2019;23(8):1985-97.

28. Michel L, Carrieri MP, Fugon L, et al. Harmful alcohol consumption and patterns of substance use in HIV-infected patients receiving antiretrovirals (ANRS-EN12-VESPA Study): relevance for clinical management and intervention. AIDS Care. 2010;22(9):1136-45.

29. Skalski LM, Watt MH, MacFarlane JC, et al. Mental health and substance use among patients in a North Carolina HIV clinic. N C Med J. 2015;76(3):148-55.

30. Starks TJ, MacDonell KK, Pennar AL, et al. Drug use among adolescents and young adults with unsuppressed HIV who use alcohol: identifying patterns of comorbid drug use and associations with mental health. AIDS Behav. 2020;24(10):2975-83.

31. Parsons JT, Starks TJ, Millar BM, Boonrai K, Marcotte D. Patterns of substance use among HIV-positive adults over 50: implications for treatment and medication adherence. Drug Alcohol Depend. 2014;139:33-40.

32. Reynolds NR. Cigarette smoking and HIV: more evidence for action. AIDS Educ Prev. 2009;21(Suppl 3):106-21.

33. Bhalla IP, Stefanovics EA, Rosenheck RA. Clinical epidemiology of single versus multiple substance use disorders: polysubstance use disorder. Med Care. 2017;55:S24-32.

34. Sandelowski M, Voils CI, Chang Y, Lee EJ. A systematic review comparing antiretroviral adherence descriptive and intervention studies conducted in the USA. AIDS Care. 2009;21(8):953-66.

35. Korthuis PT, Zephyrin LC, Fleishman JA, et al. Health-related quality of life in HIV-infected patients: the role of substance use. AIDS Patient Care STDS. 2008;22(11):859-67.

36. Baum MK, Rafie C, Lai S, et al. Alcohol use accelerates HIV disease progression. AIDS Res Hum Retroviruses. 2010;26(5):511-8.

37. DeLorenze GN, Satre DD, Quesenberry CP, Tsai AL, Weisner CM. Mortality after diagnosis of psychiatric disorders and 
co-occurring substance use disorders among HIV-infected patients. AIDS Patient Care STDS. 2010;24(11):705-12.

38. Brief DJ, Bollinger AR, Vielhauer MJ, et al. Understanding the interface of HIV, trauma, post-traumatic stress disorder, and substance use and its implications for health outcomes. AIDS Care. 2004;16(Suppl 1):S97-120.

39. Boarts JM, Sledjeski EM, Bogart LM, Delahanty DL. The differential impact of PTSD and depression on HIV disease markers and adherence to HAART in people living with HIV. AIDS Behav. 2006;10(3):253-61.

40. Cunningham CO, Sohler NL, Berg KM, Shapiro S, Heller D. Type of substance use and access to HIV-related health care. AIDS Patient Care STDs. 2006;20(6):399-407.

41. Collins LM, Lanza ST. Latent class and latent transition analysis: with applications in the social, behavioral, and health sciences. Hoboken, NJ: Wiley; 2013.

42. Gwadz MV, Collins LM, Cleland CM, et al. Using the Multiphase Optimization Strategy (MOST) to optimize an HIV care continuum intervention for vulnerable populations: a study protocol. BMC Public Health. 2017;17(1):383.

43. Mugavero MJ, Westfall AO, Cole SR, et al. Beyond core indicators of retention in HIV care: missed clinic visits are independently associated with all-cause mortality. Clin Infect Dis. 2014;59(10):1471-9.

44. Chandler RK, Kahana SY, Fletcher B, et al. Data collection and harmonization in HIV research: the seek, test, treat, and retain initiative at the National Institute on Drug Abuse. Am J Public Health. 2015;105(12):16-2422.

45. Hays RD, Spritzer KL, McCaffrey D, et al. The HIV cost and services utilization study (HCSUS) measures of health-related quality of life. Santa Monica, CA: RAND; 1998.

46. WHO Assist Working Group. The alcohol, smoking and substance involvement screening test (ASSIST): development, reliability and feasibility. Addiction. 2002;97(9):1183-94.

47. Kroenke K, Spitzer RL, Williams JB. The PHQ-9: validity of a brief depression severity measure. J Gen Intern Med. 2001;16(9):606-13.

48. Spitzer RL, Kroenke K, Williams JB, Lowe B. A brief measure for assessing generalized anxiety disorder: the GAD-7. Arch Intern Med. 2006;166(10):1092-7.

49. Prins A, Ouimette P, Kimerling R, et al. The primary care PTSD screen (PC-PTSD): development and operating characteristics. Primary Care Psychiatry. 2003;9(1):9-14.

50. Ware J Jr, Kosinski M, Keller SD. A 12-item short-form health survey: construction of scales and preliminary tests of reliability and validity. Med Care. 1996;34(3):220-33.

51. Bolck A, Croon M, Hagenaars J. Estimating latent structure models with categorical variables: one-step versus three-step estimators. Polit Anal. 2004;12(1):3-27.

52. Holm S. A simple sequentially rejective multiple test procedure. Scand Stat Theory Appl. 1979;6(2):65-70.

53. Muthen LK, Muthén L. Mplus [computer software]. CA: Los Angeles; 1998

54. R Core Team. R: A language and environment for statistical computing. Vienna: R Foundation for Statistical Computing; 2016.

55. Tsuyuki K, Pitpitan EV, Levi-Minzi MA, et al. Substance use disorders, violence, mental health, and HIV: differentiating a syndemic factor by gender and sexuality. AIDS Behav. 2017;21(8):2270-82.

56. Friedman MS, Marshal MP, Stall R, et al. Assocations between substance use, sexual risk taking and HIV treatment adherence among homeless people living with HIV. AIDS Care. 2009;21(6):692-700.
57. Wolitski RJ, Kidder DP, Fenton KA. HIV, homelessness, and public health: critical issues and a call for increased action. AIDS Behav. 2007;11(2):167-71.

58. Baxter AJ, Tweed EJ, Jatikireddi SV, Thomson H. Effects of housing first approaches on health and well-being of adults who are homeless or at risk of homelessness: systematic review and metaanalysis of randomised controlled trials. J Epidemiol Commun Health. 2019;73(5):379-87.

59. Riley ED, Vittinghoff E, Koss CA, et al. Housing first: Unsuppressed viral load among women living with HIV in San Francisco. AIDS Behav. 2019;23:2326-36.

60. Marel C, Sunderland M, Mills KL, et al. Conditional probabilities of substance use disorders and associated risk factors: progression from first use to use disorder on alcohol, cannabis, stimulants, sedatives and opioids. Drug Alcohol Depend. 2019;194:136-42.

61. McCabe SE, West BT. The 3-year course of multiple substance use disorders in the United States: a national longitudinal study. J Clin Psychiatry. 2017;78(5):e537-44.

62. Evans EA, Grella CE, Washington DL, Upchurch DM. Gender and race/ethnic differences in the persistence of alcohol, drug, and poly-substance use disorders. Drug Alcohol Depend. 2017;174:128-36.

63. Dutra L, Stathopoulou G, Basden SL, et al. A meta-analytic review of psychosocial interventions for substance use disorders. Am J Psychiatry. 2008;165(2):179-87.

64. Drake RE, O'Neal EL, Wallach MA. A systematic review of psychosocial research on psychosocial interventions for people with co-occurring severe mental and substance use disorders. J Subst Abuse Treat. 2008;34(1):123-38.

65. Priester MA, Browne T, Iachini A, et al. Treatment access barriers and disparities among individuals with co-occurring mental health and substance use disorders: an integrative literature review. J Subst Abuse Treat. 2016;61:47-59.

66. Burnam MA, Watkins KE. Substance abuse with mental disorders: specialized public systems and integrated care. Health Aff. 2006;25(3):648-58.

67. Substance Abuse and Mental Health Services Administration. Mental health and substance use disorder. https://www.samhs a.gov/find-help/disorders (2020). Accessed 5 Oct 2020.

68. Chander G, Himelhoch S, Moore RD. Substance abuse and psychiatric disorders in HIV-positive patients: epidemiology and impact on antiretroviral therapy. Drugs. 2006;66(6):769-89.

69. Stoff DM, Mitnick L, Kalichman S. Research issues in the multiple diagnoses of HIV/AIDS, mental illness and substance abuse. AIDS Care. 2004;16(Suppl 1):S1-5.

70. Soto TA, Bell J, Pillen M, For The HIV/AIDS Treatment Adherence Health Outcomes Cost Study Group. Literature on integrated HIV care: a review. AIDS Care. 2004;16(Suppl 1):43-55.

71. Weaver MR, Conover CJ, Proescholdbell RJ, et al. Utilization of mental health and substance abuse care for people living with HIV/AIDS, chronic mental illness, and substance abuse disorders. J Acquir Immune Defic Syndr. 2008;47(4):449-58.

72. Kraemer KL, McGinnis KA, Fiellin DA, et al. Low levels of initiation, engagement, and retention in substance use disorder treatment including pharmacotherapy among HIV-infected and uninfected veterans. J Subst Abuse Treat. 2019;103:23-32.

73. Calsyn RJ, Klinkenberg WD, Morse GA, et al. Recruitment, engagement, and retention of people living with HIV and cooccurring mental health and substance use disorders. AIDS Care. 2004;16(Suppl 1):S56-70.

74. Mueser KT, Gingerich S. Treatment of co-occurring psychotic and substance use disorders. Soc Work Public Health. 2013;28(3-4):424-39.

75. Ciketic S, Hayatbakhsh MR, Doran CM, Najman JM, McKetin R. A review of psychological and pharmacological 
treatment options for methamphetamine dependence. J Subst Use. 2011;17(4):363-83.

76. Durvasula R, Miller TR. Substance abuse treatment in persons with HIV/AIDS: challenges in managing triple diagnosis. Behav Med. 2014;40(2):43-52.

77. Van Wormer R, Van Wormer K. Non-abstinence-based supportive housing for persons with co-occurring disorders: a human rights perspective. J Progress Hum Serv. 2009;20(2):152-65.

78. Vakharia SP, Little J. Starting where the client is: harm reduction guidelines for clinical social work practice. Clin Soc Work J. 2016;45(1):65-76.

79. Byrd KK, Hardnett F, Hou JG, et al. Improvements in retention in care and HIV viral suppression among persons with HIV and comorbid mental health conditions: patient-centered HIV care model. AIDS Behav. 2020;24(12):3522-32.

80. Fiellin DA, Weiss L, Botsko M, et al. Drug treatment outcomes among HIV-infected opioid-dependent patients receiving buprenorphine/naloxone. J Acquir Immune Defic Syndr. 2011;56(Suppl 1):S33-8.

81. Springer SA, Chen S, Altice FL. Improved HIV and substance abuse treatment outcomes for released HIV-infected prisoners: the impact of buprenorphine treatment. J Urban Health. 2010;87(4):592-602.

82. Fanucchi L, Springer SA, Korthuis PT. Medications for treatment of opioid use disorder among persons living with HIV. Curr HIV/ AIDS Rep. 2019;16(1):1-6.

83. Low AJ, Mburu G, Welton NJ, et al. Impact of opioid substitution therapy on antiretroviral therapy outcomes: a systematic review and meta-analysis. Clin Infect Dis. 2016;63(8):1094-104.

84. Lyss SB, Buchacz K, McClung RP, Asher A, Oster AM. Responding to outbreaks of human immunodeficiency virus among persons who inject drugs-United States, 2016-2019: perspectives on recent experience and lessons learned. J Infect Dis. 2020;222(Supp 5):S239-49.

85. Mattick RP, Breen C, Kimber J, Davoli M. (2014) Buprenorphine maintenance versus placebo or methadone maintenance for opioid dependence. Cochrane Database Syst Rev. 2014;2:CD002207.

86. Spire B, Lucas GM, Carrieri MP. Adherence to HIV treatment among IDUs and the role of opioid substitution treatment (OST). Int J Drug Policy. 2007;18(4):262-70.

87. Wakeman SE, Larochelle MR, Ameli O, et al. Comparative effectiveness of different treatment pathways for opioid use disorder. JAMA Netw Open. 2020;3(2):e1920622.
88. Grella CE, Ostile E, Scott CK, Dennis M, Carnavale J. A scoping review of barriers and facilitators to implementation of medications for treatment of opioid use disorder within the criminal justice system. Int J Drug Policy. 2020;81:102768.

89. Michels II, Stover H. Harm reduction-from a conceptual framework to practical experience: the example of Germany. Subst Use Misuse. 2012;47(8-9):910-22.

90. Feldman MB, Thomas JA, Alexy ER, Irvine MK. Crystal methamphetamine use and HIV medical outcomes among HIV-infected men who have sex with men accessing support services in New York. Drug Alcohol Depend. 2015;147:266-71.

91. Goodman-Meza D, Shoptaw S, Weiss RE, et al. Methamphetamine use drives decreases in viral suppression for people living with HIV released from a large municipal jail: results of the LINK LA clinical trial. Drug Alcohol Depend. 2019;202:178-84.

92. Rajasingham R, Mimiaga MJ, White JM, et al. A systematic review of behavioral and treatment outcome studies among HIVinfected men who have sex with men who abuse crystal methamphetamine. AIDS Patient Care STDS. 2012;26(1):36-52.

93. Wechsberg WM, Golin C, El-Bassel N, Hopkins J, Zule W. Current interventions to reduce sexual risk behaviors and crack cocaine use among HIV-infected individuals. Curr HIV/AIDS Rep. 2012;9(4):385-93.

94. Rose ME, Grant JE. Pharmacotherapy for methamphetamine dependence: a review of the pathophysiology of methamphetamine addiction and the theoretical basis and efficacy of pharmacotherapeutic interventions. Ann Clin Psychiatry. 2008;20(3):145-55.

95. Crane HM, Nance RM, Whitney BM, et al. Drug and alcohol use among people living with HIV in care in the United States by geographic region. AIDS Care. 2021. https://doi.org/10.1080/09540 121.2021.1874274.

96. Gardner LI, Marks G, Shahani L, et al. Assessing efficacy of a retention-in-care intervention among HIV patients with depression, anxiety, heavy alcohol consumption and illicit drug use. AIDS. 2016;30(7):1111-9.

Publisher's Note Springer Nature remains neutral with regard to jurisdictional claims in published maps and institutional affiliations. 